Document downloaded from:

http://hdl.handle.net/10251/100443

This paper must be cited as:

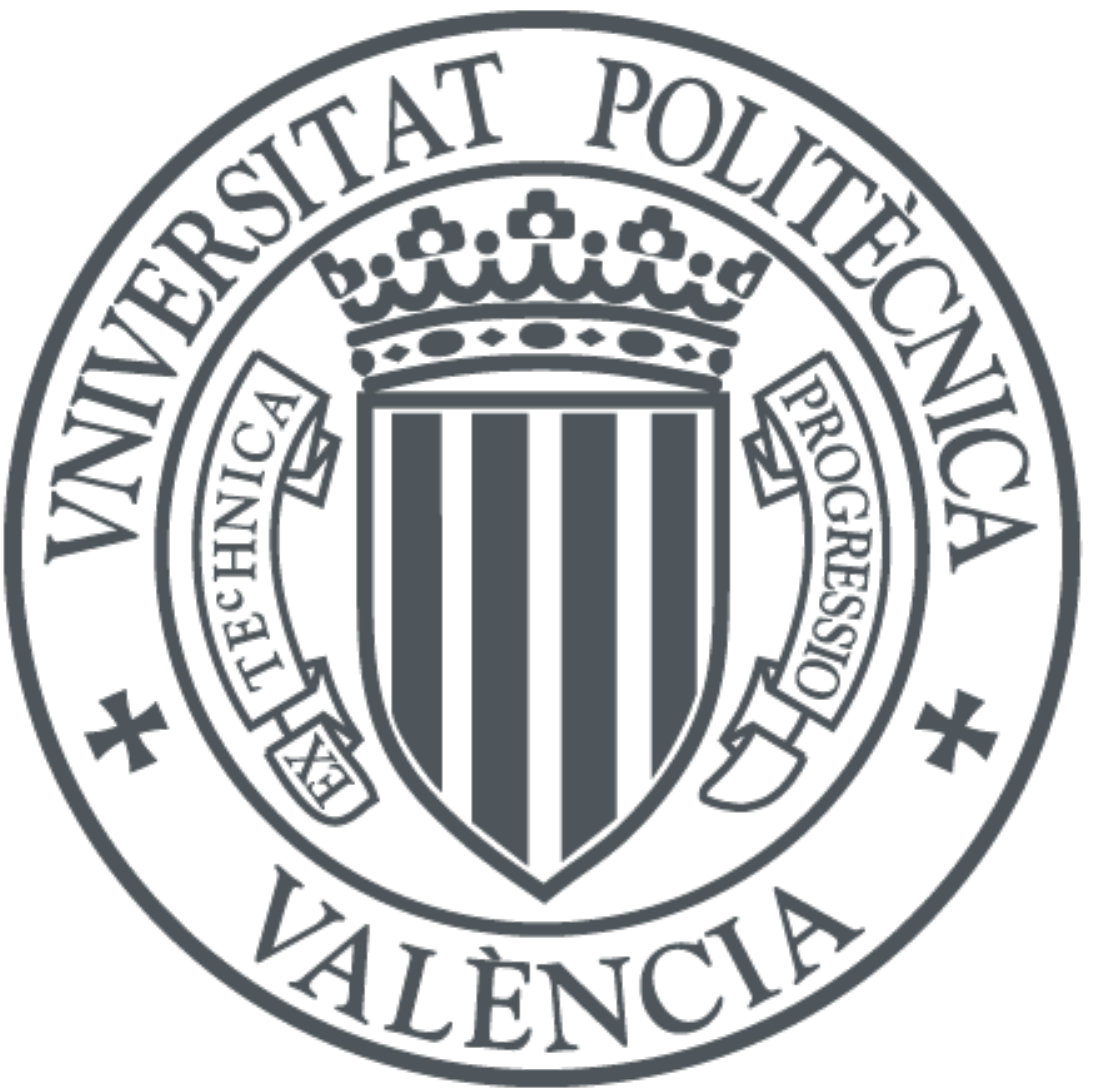

The final publication is available at

http://dx.doi.org/10.1016/j.jmmm.2017.08.041

Copyright Elsevier

Additional Information 


\title{
In-plane omnidirectional magnetic field sensor based on Giant Magneto Impedance (GMI)
}

\author{
Ana Díaz-Rubio ${ }^{1,4}$, Héctor García-Miquel ${ }^{1,2,3}$, Víctor Manuel García-Chocano ${ }^{1}$ \\ ${ }^{1}$ Department of Electronic Engineering, Universitat Politècnica de València, C/Camino de Vera s/n, E-46022 \\ Valencia, Spain. \\ ${ }^{2}$ ITEAM Research Institute, Universitat Politècnica de València, C/Camino de Vera s/n, E-46022 Valencia, Spain. \\ ${ }^{3}$ TFM Group, Department of Physics, University of Cambridge, CB3 OHE, UK \\ ${ }^{4}$ Department of Electronics and Nanoengineering, Aalto University, P. O. Box 15500, FI-00076 Aalto, Finland.
}

\begin{abstract}
In this work the design and characterization of an omnidirectional in-plane magnetic field sensor are presented. The sensor is based on the Giant Magnetoimpedance (GMI) effect in glass-coated amorphous microwires of composition $\left(\mathrm{Fe}_{6} \mathrm{Co}_{94}\right)_{72.5} \mathrm{Si}_{12.5} \mathrm{~B}_{15}$. For the first time, a circular loop made with a microwire is used for giving omnidirectional response. In order to estimate the GMI response of the circular loop we have used a theoretical model of GMI, determining the GMI response as the sum of longitudinal sections with different angles of incidence. As a consequence of the circular loop, the GMI ratio of the sensor is reduced to $15 \%$ instead of $100 \%$ for the axial GMI response of a microwire. The sensor response has been experimentally verified and the GMI response of the circular loop has been studied as function of the magnetic field, driven current, and frequency. First, we have measured the GMI response of a longitudinal microwire for different angles of incidence, covering the full range between the tangential and perpendicular directions to the microwire axis. Then, using these results, we have experimentally verified the decomposition of a microwire with circular shape as longitudinal segments with different angles of incidence. Finally, we have designed a signal conditioning circuit for the omnidirectional magnetic field sensor. The response of the sensor has been studied as a function of the amplitude of the incident magnetic field.
\end{abstract}

Keywords: Giant Magneto Impedance (GMI), amorphous microwire, magnetic field sensor. 


\section{Introduction}

Glass coated amorphous microwires have been intensively studied due to its outstanding magnetic properties, as giant magnetoimpedance (GMI) [1-9] or its absorption properties at microwave frequencies [10-19]. The GMI effect is related to the change of the skin depth on the microwire due to strong changes of the dynamic circular permeability [20,21]. In particular, the microwires studied in this work are of composition $\left(\mathrm{Fe}_{6} \mathrm{Co}_{94}\right)_{72.5} \mathrm{Si}_{12.5} \mathrm{~B}_{15}$ which has negative but close to zero magnetostriction $\left(\lambda_{\mathrm{S}}=-10^{-7}\right)$ [22]. Due to the fabrication process, they present a shell of circular permeability with a "bamboo like" structure and an inner core with axial anisotropy [23]. Since the microwire is amorphous, the domain structure is a consequence of the shape and magnetoelastic anisotropies resulting from the stress caused by the fabrication process. The microwires with the aforementioned composition present a circular anisotropy characterized by an anisotropy filed $\mathrm{H}_{\mathrm{a}}=285 \mathrm{~A} / \mathrm{m}$ [24]. When an external magnetic field $\mathrm{H}_{0}$ is applied in the axial direction of the microwire, the effective dynamic circular permeability reaches a maximum as the applied field approach to the anisotropy field. In this point the skin depth shows a minimum and therefore the impedance is maximized $[20,25]$.

The GMI effect has special application in the development of highly sensitive magnetic sensors $[5,6,26,27]$ and current sensors [28]. Mohri et al. have done a remarkable contribution in the development of electronic circuits for GMI sensors [29,30]. All GMI sensors reported in previous works were done with the microwire placed longitudinally, and therefore the sensibility of the GMI depends on the angle between the applied magnetic field and the axis of the microwire. In this paper, we present an inplane omnidirectional magnetic field sensor based on a microwire in the shape of a circular loop (see Fig.6(a)), and we have done theoretical simulations, experimental measurements and the signal conditioning circuit.

The microwires employed in this work have been studied in a previous work of the authors [24], where GMI measurements were performed with AC current and frequency as parameters, and a signal conditioning circuit was developed in order to design a magnetic field sensor. In this work we extend this study to a circular loop geometry of the microwire. For a proper understanding of the proposed configuration, a phenomenological model for circular microwires is presented. The results obtained are supported by a set of experimental measurements and the development of a high-sensitivity sensor.

\section{Theoretical approach for GMI in circular microwires}

The magnetic domain structure of microwires is rather complex and hinders the development of precise theoretical models. This fact is basically due to the change of magnetization with the radius because of the shape and magnetoelastic anisotropies, the latter being due to the radial variation of the stress in the microwire. Such a stress is caused by the radial gradient of temperature during the fabrication process and the different expansion coefficient of the glass shield and the inner ferromagnetic core. Many efforts have been made to model the GMI effect in microwires and many remarkable works have been reported [5,20,21,25,31-33], but indeed any of them gets a perfect agreement with experimental measurements. The discrepancies are especially important at zero magnetic field, where low levels of impedance are predicted while experimental data show a value close to the values found in saturation (i.e. at high magnetic fields).

Our aim is to present a simple approach that models the behaviour of a circular microwire. For this purpose, we will assume the loop can be decomposed in a series of small sections of microwires excited at different angles with respect to the external magnetic field. Under this assumption, we can discompose the incident magnetic field in two orthogonal components, one axial to the wire and the other perpendicular, the latter playing a minor role in the impedance and therefore being negligible. Thereby the problem is reduced to the study of a straight microwire excited with an external magnetic field at a given angle.

The GMI in a microwire depends on the dynamics of the magnetization due to the interaction of the external magnetic field and the circular field produced inside the microwire due to the AC current flowing through it. The movement of the magnetization with an applied magnetic field along with the Maxwell equations are described by the Landau-LifshiftGilbert (LLG) equation:

$\frac{\partial \vec{M}}{\partial t}=-\mu_{o} \gamma(\vec{M} \times \vec{H})+\alpha\left(\vec{M} \times \frac{\partial \vec{M}}{\partial t}\right)$

where $\gamma$ is the gyromagnetic ratio, $\mu_{0}$ the permeability of free space, $\alpha$ a damping parameter, $M_{s}$ the saturation magnetization, $H=H_{0}+h_{a c}$ the total magnetic field (static and 
dynamic), and $M=M_{s}+m_{a c}$ the total magnetization. Applying the LLC equation, the corresponding boundary conditions and taking into account the two excitations (external magnetic field and the ac circular magnetic field produced by current flowing through the microwire) the dynamic permeability and the impedance can be obtained [20,32,33]. The GMI is related to the change in the skin depth due to changes of the dynamic transversal permeability. The surface impedance of the microwire $Z_{S}(\omega)$ can be expressed through the classical analysis of a cylindrical conductor, and thereby the impedance of the microwire as $Z(\omega)=Z_{S}(\omega) l$, being $l$ the microwire's length. Although the impedance is a tensor, we are only interested in the longitudinal impedance $Z_{Z Z}(\omega)$.

$Z(\omega)=R_{D C} \frac{\beta a}{2} \frac{J_{0}(\beta a)}{J_{1}(\beta a)}$

$\beta=\frac{(1-j)}{\delta}=(1-j) \sqrt{\pi f \mu_{\phi} \sigma}$

$\delta=\frac{1}{\sqrt{\pi f \mu_{\phi} \sigma}}$

$R_{D C}=\frac{1}{\sigma} \frac{l}{\pi a^{2}}$

$\mathrm{R}_{\mathrm{DC}}$ being the $\mathrm{DC}$ resistance of the microwire, $\sigma$ the conductivity, $a$ the radius, $\beta$ the wave vector, $f$ the frequency, $\mu_{\phi}$ the transversal permeability, $J_{n}(x)$ the Bessel functions of $n$ th order and $\delta$ the skin depth.

The magnetic microwire is amorphous and its magnetization is determined by the shape anisotropy and the magneto-elastic anisotropy. In order to determine the direction of the magnetization when an external magnetic field is applied we consider the minimization of energy, U through the StonerWohlfarth model:

$U=-\mu_{0} M_{S}\left(0.5 H_{a} \cos (90-\theta)+H_{O} \cos \theta\right)$

where $H_{0}$ is the external field, $H_{a}$ the anisotropy field (with $\mathrm{H}_{\mathrm{a}}$ $=2 \mathrm{~K} / \mu_{0} \mathrm{M}_{\mathrm{S}}$, $\mathrm{K}$ being the effective anisotropy constant) and $\theta$ the angle between the axis of the microwire and the magnetization direction (see Fig.1(a)). We suppose a circular transversal anisotropy in the microwire with a "bamboo like" domain structure $\left(\alpha=90^{\circ}\right)$ as illustrated in Fig.1(b). With the minimization of the energy two solutions are obtained: $\theta=90^{\circ}$ for $H_{o} \geq H_{a}$, and $\theta=\operatorname{arcos}\left(H_{o} / H_{a}\right)$ for $H_{o} \leq H_{a}$. By solving the LLG equation and Maxwell equations we get the effective transverse susceptibility [30,31]:

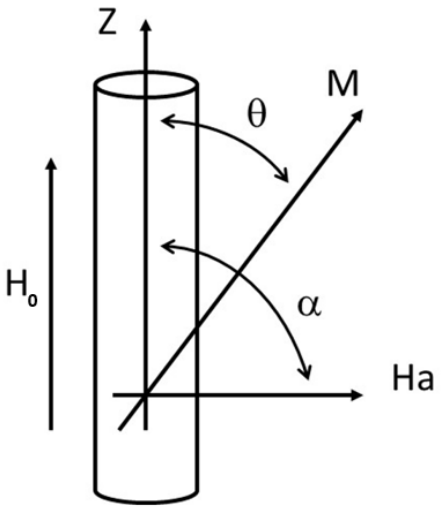

(a)

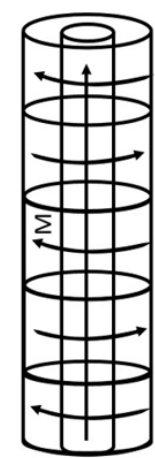

(b)
Fig.1: (a) Specification of the angles between the wire axis and the magnetization $(\theta)$ and anisotropy field $(\alpha)$. (b) Domain structure with an inner core with axial magnetization and outer layer with circular magnetization with a "bamboo like" configuration.

$$
\begin{gathered}
\chi_{e f f, \phi}(\omega)=\frac{\omega_{M}^{2}+\omega_{M}\left(\omega_{2}-j \omega \alpha\right)}{\left(\omega_{1}-j \omega \alpha\right)\left(\omega_{2}+\omega_{M}-j \omega \alpha\right)-\omega^{2}} \\
\omega_{1}=\mu_{0} \gamma\left[H_{0} \cos \theta+H_{a} \cos 2(90-\theta)\right]
\end{gathered}
$$

$\omega_{2}=\mu_{0} \gamma\left[H_{0} \cos \theta+H_{a} \cos ^{2}(90-\theta)\right]$

$$
\omega_{M}=\mu_{0} \gamma M_{S}
$$

Applying the Maxwell's equations and the boundary conditions we obtain the longitudinal impedance of the microwire, which for the high frequency regime has the form [20,31-33]:

$$
\begin{array}{r}
Z_{z z}(\omega)=\frac{(1+j)}{2 \pi a} \sqrt{\frac{\pi f \mu_{0}}{\sigma}}\left(\sin ^{2} \theta+\right. \\
\left.\sqrt{\left(1+\chi_{e f f, \phi}\right)} \cos ^{2} \theta\right)
\end{array}
$$

Nevertheless, this solution presents some discrepancies in the zone of zero magnetic field where the predicted impedance tends to very low values (see Fig. 2). This behaviour contrasts with measured data previously reported for this kind of microwires [24]. To overcome this issue, we consider a simple model based on the application of Eq. 2 with the susceptibility given by Eq. 7. It must be noted that Eq.2 for the high frequency limit is equal to Eq.11 with $\theta=0^{\circ}$. This can be understood considering that in the magnetic field around zero, the skin depth is big enough to consider that the high frequency 
magnetic field is penetrating through all the wire, being the most important contribution to the impedance that of the inner core with axial magnetization. It is also important to remark that we take as an approximation the magnetic permeability as a scalar instead of a tensor [13].

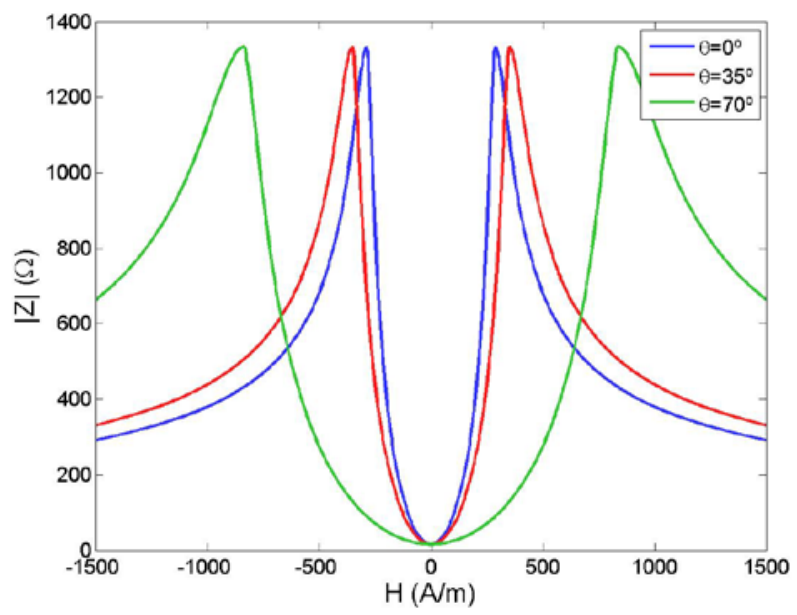

Fig.2: Simulation of GMI with the solution in Eq.11., with parameters, $\mathrm{a}=3.8 \mu \mathrm{m}, \mathrm{f}=15 \mathrm{MHz}, \mathrm{l}=3.3 \mathrm{~cm}, \sigma=8.7 \cdot 10^{5} \mathrm{~S} / \mathrm{m}$, $\mu_{0} \mathrm{M}_{\mathrm{S}}=0.5 \mathrm{~T}, \alpha=0.15, \mathrm{Ha}=285 \mathrm{~A} / \mathrm{m}$ and $\gamma=19.36 \cdot 10^{10} \mathrm{~T}^{-1} \mathrm{~s}^{-1}$.

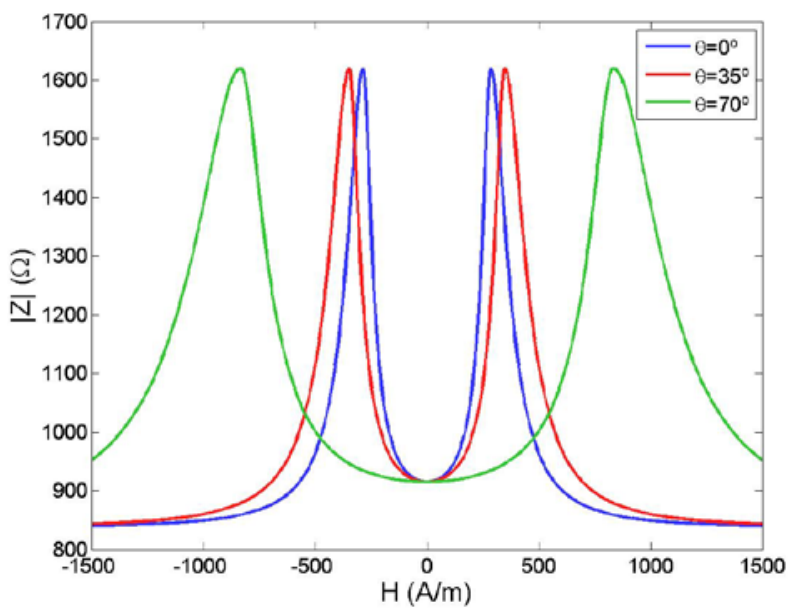

Fig.3: Impedance of a microwire calculated with Eqs. 2 and 7 and parameters $\mathrm{a}=3.8 \mu \mathrm{m}, \mathrm{f}=15 \mathrm{MHz}, \mathrm{l}=3.3 \mathrm{~cm}, \sigma=8.7 \cdot 10^{5} \mathrm{~S} / \mathrm{m}$, $\mu_{0} \mathrm{M}_{\mathrm{S}}=0.5 \mathrm{~T}, \alpha=0.15, \mathrm{Ha}=285 \mathrm{~A} / \mathrm{m}$ and $\gamma=19.36 \cdot 10^{10} \mathrm{~T}^{-1} \mathrm{~s}^{-1}$.

As shown in Fig. 3, this simple approach gets a closer fit to the experimental measurements exposed in the next section. The error around zero magnetic field has been improved, getting the expected value of the experimental data. Figure 4 describes the response of the skin depth $\delta$ with the external magnetic field. As expected, the value of $\delta$ is minimized at certain values of magnetic field where the projection of the field reaches the anisotropy field $\mathrm{H}_{\mathrm{a}}$. Thus the impedance of the microwire is maximum at these points since the effective section of the microwire is constrained by the skin-depth effect. At higher fields $\delta$ increases and therefore the impedance is reduced. When its value is much higher than the radius of the microwire, the sample is saturated and its impedance experience small variations with the external field.

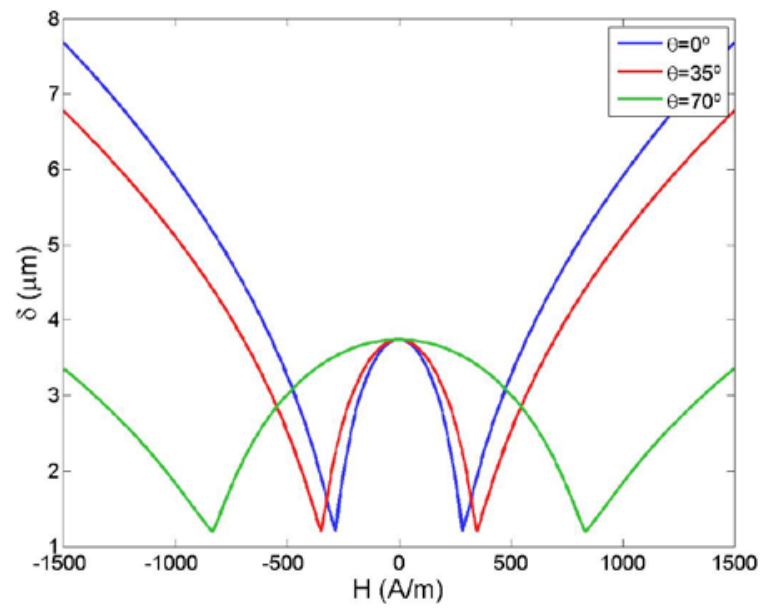

Fig.4: Simulation of the skin depth in a microwire with parameters $\mathrm{a}=3.8 \mu \mathrm{m}, \mathrm{f}=15 \mathrm{MHz}, \mathrm{l}=3.3 \mathrm{~cm}, \sigma=8.7 \cdot 10^{5} \mathrm{~S} / \mathrm{m}$, $\mu_{0} \mathrm{M}_{\mathrm{S}}=0.5 \mathrm{~T}, \alpha=0.15, \mathrm{Ha}=285 \mathrm{~A} / \mathrm{m}$ and $\gamma=19.36 \cdot 10^{10} \mathrm{~T}^{-1} \mathrm{~s}^{-1}$.

As previously introduced, the circular loop configuration of the microwire (see Fig.6(a)) can be thought as a superposition of longitudinal elements with different incident angles. The model previously introduced can be used to calculate the angular-dependent impedance $Z_{s}(\theta)$ of a microwire. Thus, the total impedance of a loop can be calculated by integrating the contribution of the different elements:

$Z_{\text {Loop }}=\int_{0}^{2 \pi} Z(\theta) d \theta$

In Fig. 5 it is shown the impedance of a microwire with the shape of a circular loop. It has been calculated by taking linear segments of microwires with angular steps of $1^{\circ}$. It is found that the response of a circular microwire presents the characteristic shape with two peaks, although these peaks are wider than those found for the reference case of a straight sample and with an incident angle of $0^{\circ}$. In addition, the GMI ratio is lower and the position of the peaks are slightly higher. 


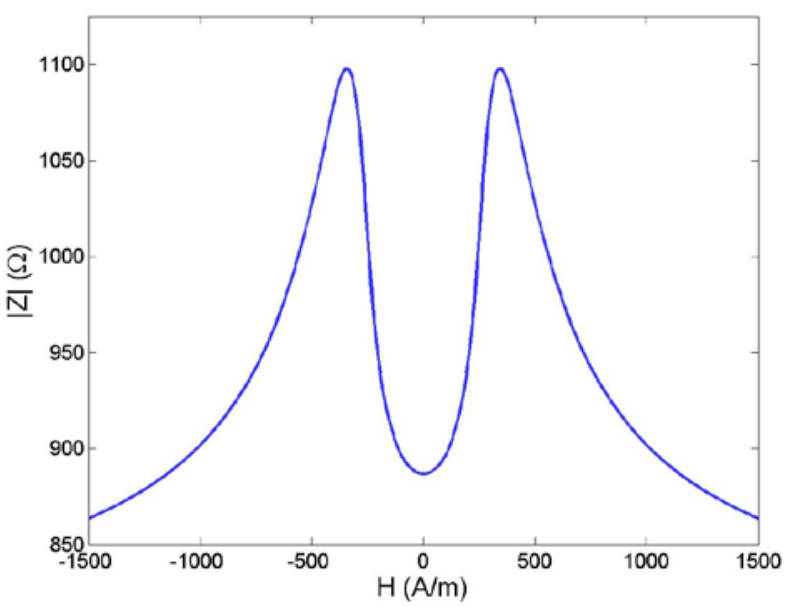

Fig. 5: Simulation of GMI of a microwire loop with $0.5 \mathrm{~cm}$ of radius. The response is obtained through the sum of the contributions of GMI of pieces of microwires in steps of $1^{\circ}$, with parameters $a=3.8 \mu \mathrm{m}, \mathrm{f}=15 \mathrm{MHz}, \sigma=8.7 \cdot 10^{5} \mathrm{~S} / \mathrm{m}, \mu_{0} \mathrm{M}_{\mathrm{S}}$ $=0.5 \mathrm{~T}, \alpha=0.25, \mathrm{Ha}=285 \mathrm{~A} / \mathrm{m}$ and $\gamma=19.36 \cdot 10^{10} \mathrm{~T}^{-1} \mathrm{~s}^{-1}$.

\section{Experimental results and analysis.}

Figure 6(b) shows a SEM image of the microwires here employed, where the ferromagnetic core as well as the glass coat can be clearly distinguished. The characterization of the GMI phenomena in microwires is usually performed by measuring the change of impedance versus magnetic field for a given frequency and a constant AC amplitude of the driving current. The driving current generates a circular magnetic field into the sample that affects to its magnetization processes. The GMI effect is measured at constant current amplitude in order to keep a constant contribution of the AC magnetic field.
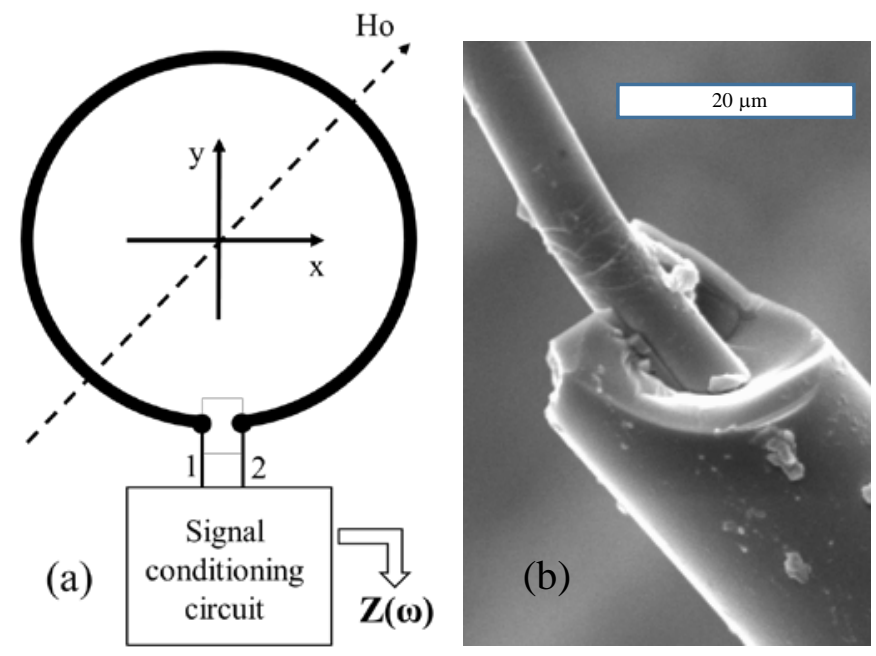

Fig. 6: (a) Schematic of the microwire in a circular shape to measure the in plane (x-y) omnidirectional magnetic field $\mathrm{H}_{0}$, (b) SEM image of a magnetic microwire. The diameter of the ferromagnetic core is $3.8 \mu \mathrm{m}$ and thickness of glass coat is $8 \mu \mathrm{m}$.

A straight sample of $3.3 \mathrm{~cm}$ has been experimentally characterized. Incident fields at different angles $\theta$ with respect to the axis of the sample have been applied. We have used the experimental setup previously employed in [24]. First, the impedance has been characterized for a constant AC current of $1 \mathrm{~mA}_{\mathrm{p}}$, frequency of $15 \mathrm{MHz}$ and incident angles from $\theta=0^{\circ}$ to $\theta=90^{\circ}$ in a $15^{\circ}$ step. The experimental results are summarized in Fig. 7. When the magnetic field is parallel to the microwire $\left(\theta=0^{\circ}\right)$, the impedance response shows doublepeak behaviour (typical response when the wire has circular anisotropy with two maxima appearing at the anisotropy field $\mathrm{Ha}=285.4 \mathrm{~A} / \mathrm{m}$. Similar results were obtained in [24] where the same microwire composition was employed. The position of the peak of GMI increases with the increase of the incident angle of the magnetic field. Note that the maximum value of the impedance presents small variations for a broad range of angles. However, when the magnetic field impinges perpendicular to the microwire direction $\left(\theta=90^{\circ}\right)$, the GMI response disappears.

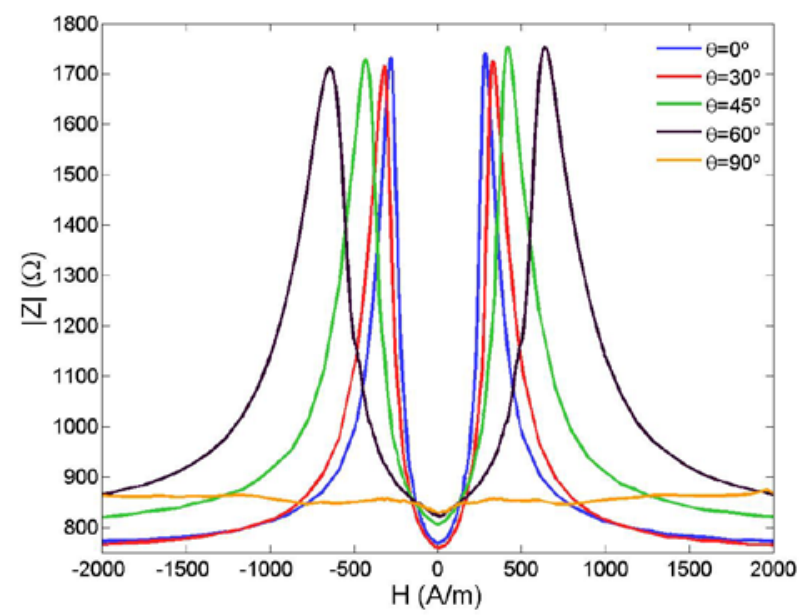

Fig. 7: Impedance modulus of a longitudinal microwire of $3.3 \mathrm{~cm}$ as a function of the incident angle of the magnetic field $(\theta)$, with a constant current of $1 \mathrm{mAp}$ and $15 \mathrm{MHz}$.

The behaviour of the impedance with the incident angle can be understood as the superposition of the parallel and perpendicular projections of the magnetic field with respect to the microwire (see inset in Figure 8). Due to the negligible GMI response of the perpendicular projection, the displacement of the peak position $\left(\mathrm{H}_{\mathrm{k}}\right)$ is mainly controlled by 
the projection of the applied field in the axial direction of the microwire. Using this approach, the peak position can be determined by $H_{k}=\mathrm{Ha} / \cos (\theta)$, where Ha is the anisotropy field. Figure 8 shows a comparison between the measured values of $\mathrm{H}_{\mathrm{k}}$ and the prediction obtained using the projection of the field on the axial direction of the microwire.

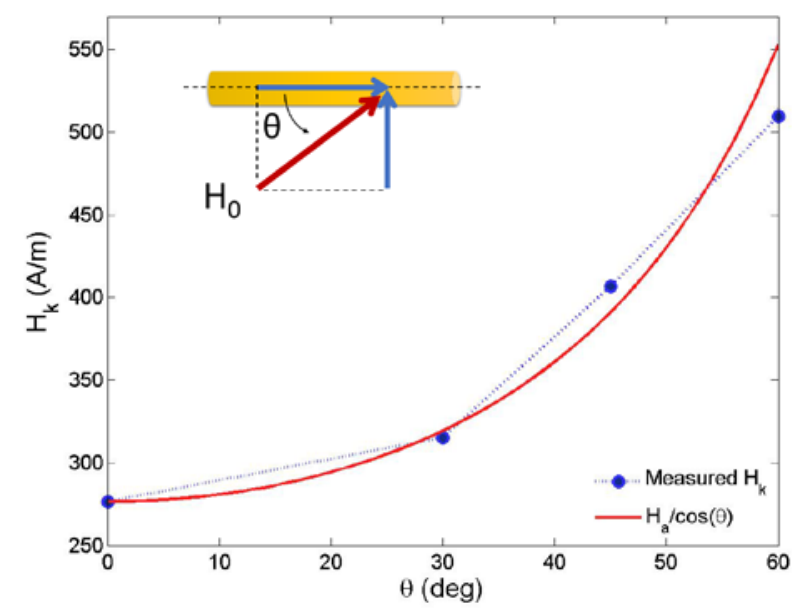

Fig. 8: Magnetic field where the maximum of impedance is found $\left(\mathrm{H}_{\mathrm{k}}\right)$ as a function of the incident angle $(\theta)$. Comparison with the theoretical value obtained by using the projection over the wire direction.

Following this procedure, the complete GMI response at a certain incident angle can be obtained as the superposition of the parallel and perpendicular projections, i.e. $Z(\theta)=Z_{0} \cos (\theta)$ $+Z_{90} \sin (\theta)$, although the perpendicular contribution in the magnetic field range under study can be neglected. A particular case for $\theta=45^{\circ}$ is calculated and represented in Figure 9. In this figure, we can see the comparison between the measured response at $45^{\circ}$ and the modelled response calculated using the aforementioned projection. As we obtain a good agreement in the comparison we are going to use this model for the simulation of the impedance of a microwire in the shape of a circular loop. In Fig.10 there is the comparison between the measured microwire in a circular loop and the impedance calculated by the sum of the GMI for angles between $0^{\circ}$ to $90^{\circ}$ in steps of $15^{\circ}$. As it can be seen in Fig.10 the model used fits very well with the experimental measurement for the circular loop sensor.

Figure 11 shows the measurements of the impedance of a microwire in the shape of a circular loop with $0.5 \mathrm{~cm}$ of radius for different AC currents flowing through it. It is found that the anisotropy field increases with decreasing currents. The excitation of the microwire at lower currents leads to higher GMI peaks, although for currents below $5 \mathrm{~mA}$ there is more noise due to the magnetization processes. The noise is probably due to wall movements that leads to Barkhausen jumps. With a current of $7 \mathrm{~mA}$ the measurements exhibit lower levels of noise and a monotonous variation of impedance without perceptible Barkhausen jumps. Regarding these data, we conclude that the sensor presented in the next section should be excited at $7 \mathrm{~mA}_{\mathrm{p}}$. This current reduces the noise and offers a high sensitivity in the low field region.

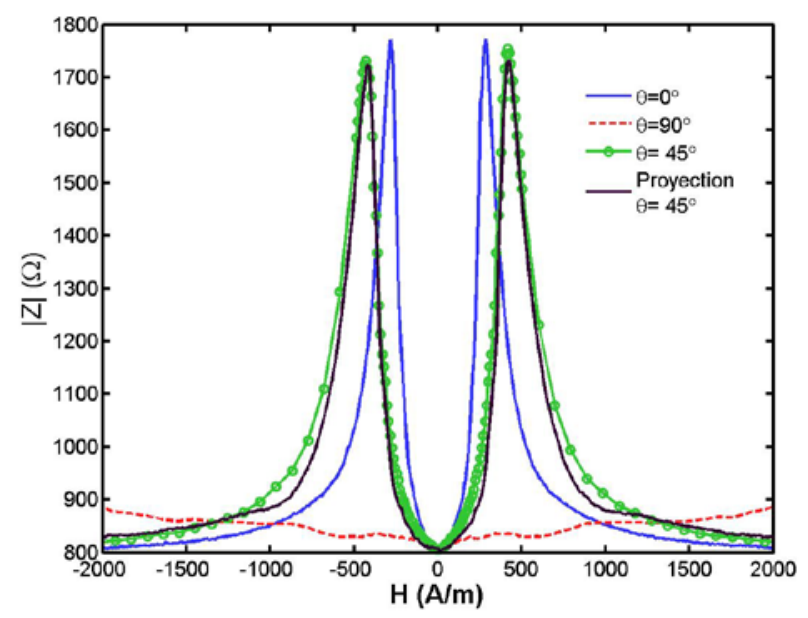

Fig. 9: Superposition model and comparison with the measurement results for $\theta=45$ with a constant current of 1 mAp and $15 \mathrm{MHz}$.

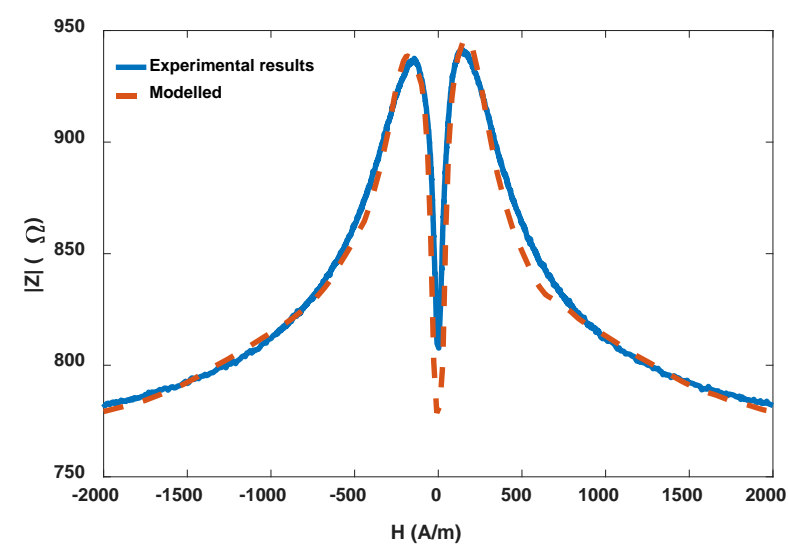

Fig. 10: Impedance response for the circular loop with a constant current of $7 \mathrm{mAp}$ and $11 \mathrm{MHz}$. Comparison between the measurement (dotted blue line) and the model based on measured longitudinal elements (solid red line). The model uses the measurements of GMI form $0^{\circ}$ to $90^{\circ}$ in steps of $15^{\circ}$. 


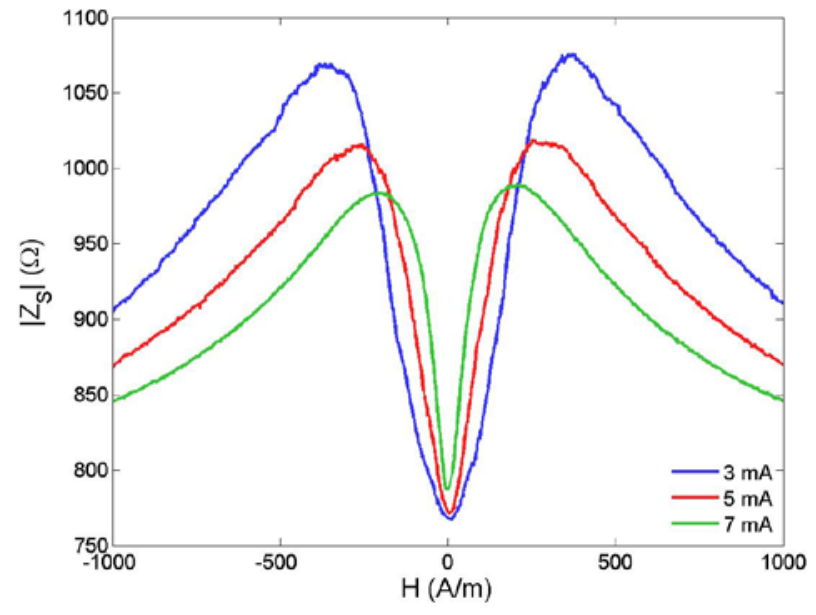

Fig. 11: Impedance modulus of a circular loop microwire with $0.5 \mathrm{~cm}$ of radius as a function of the current and $15 \mathrm{MHz}$ frequency.

The impedance of the microwire as a function of the frequency is shown in Fig. 12. According to previous results [24], the GMI effect is stronger at higher frequencies. For this reason, the proposed sensor will operate at the maximum measured current, that is, $15 \mathrm{MHz}$.

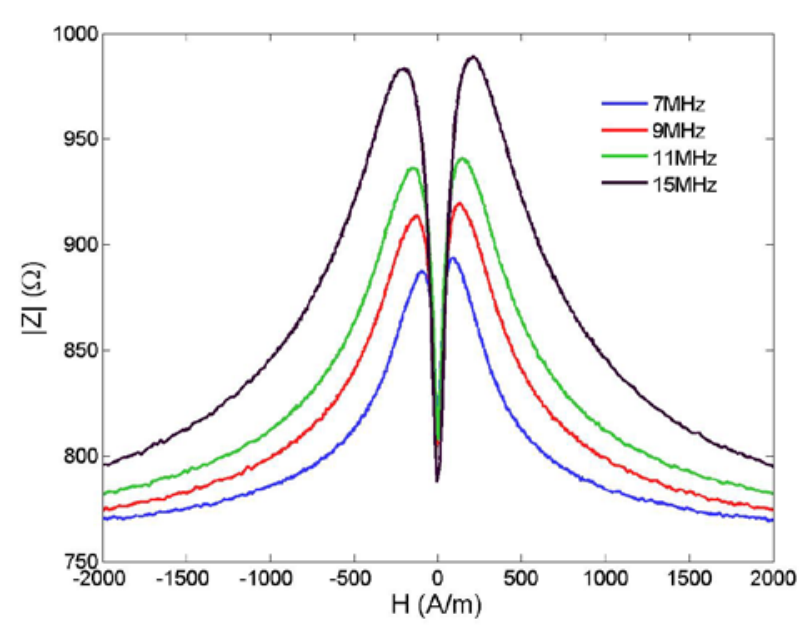

Fig. 12: Impedance modulus of a circular loop microwire with $0.5 \mathrm{~cm}$ of radius for a flowing current of $7 \mathrm{mAp}$.

\section{Signal conditioning.}

The proposed conditioning circuit is shown in Fig. 13. First, a sinusoidal signal at $15 \mathrm{MHz}$ is generated in a Colpitts oscillator. This signal is buffered and driven to a current source that feeds the microwire with a constant AC current. The voltage of the microwire pass through an envelope detector in order to measure its AC amplitude. Finally, the resulting signal goes to an amplifier with a low pass filter and offset adjustment.

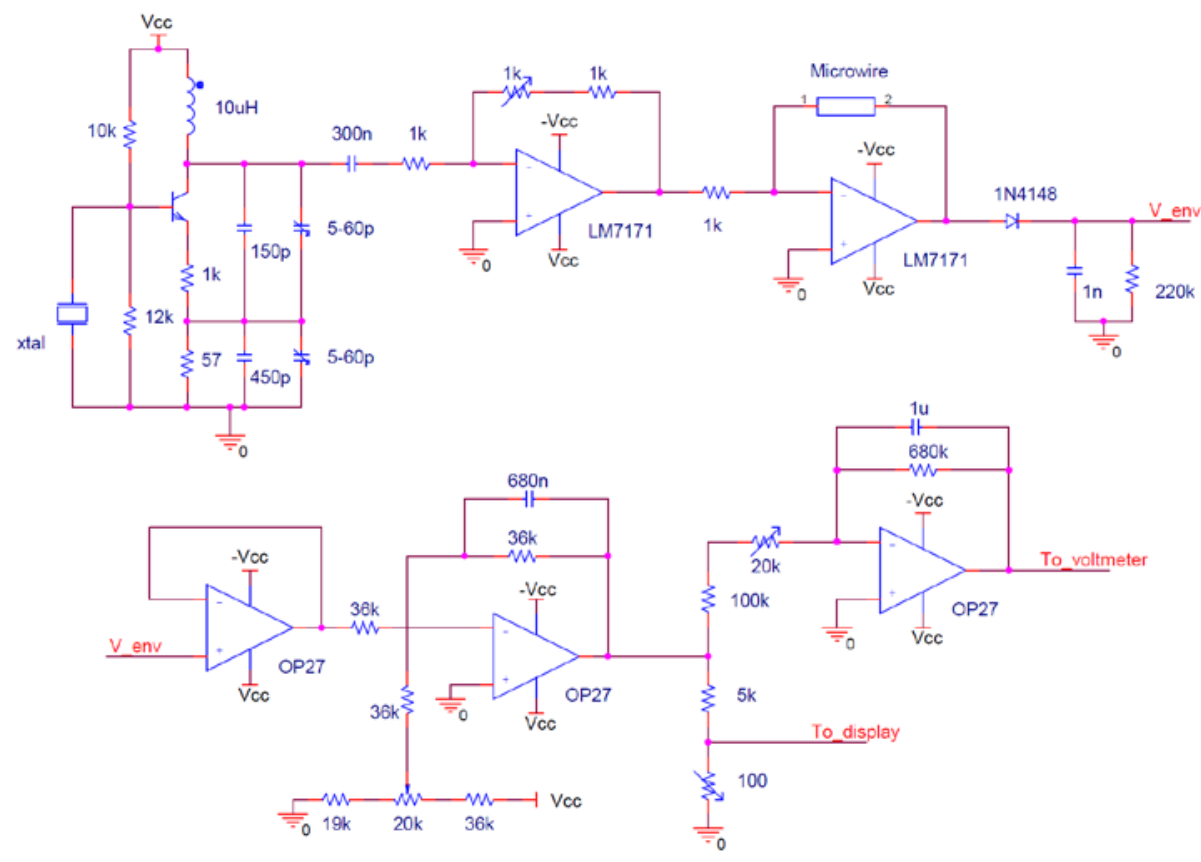

Fig. 13: Schematic of the signal conditioning circuit designed for the GMI sensor. 
The circuit of Fig. 13 has been built and characterized. A photograph of the resulting board can be observed in Fig. 14. Its response to an external magnetic field is shown in Fig. 15 and exhibits a usable response in the range of $\pm 200 \mathrm{~A} / \mathrm{m}$. Since the response is monotonic within this range, it is suitable to be digitally corrected in order to get a linear response between magnetic field and output voltage. Note that, since the sensor is omnidirectional, it cannot distinguish de direction of the applied field but only the in-plane magnitude of the magnetic field.

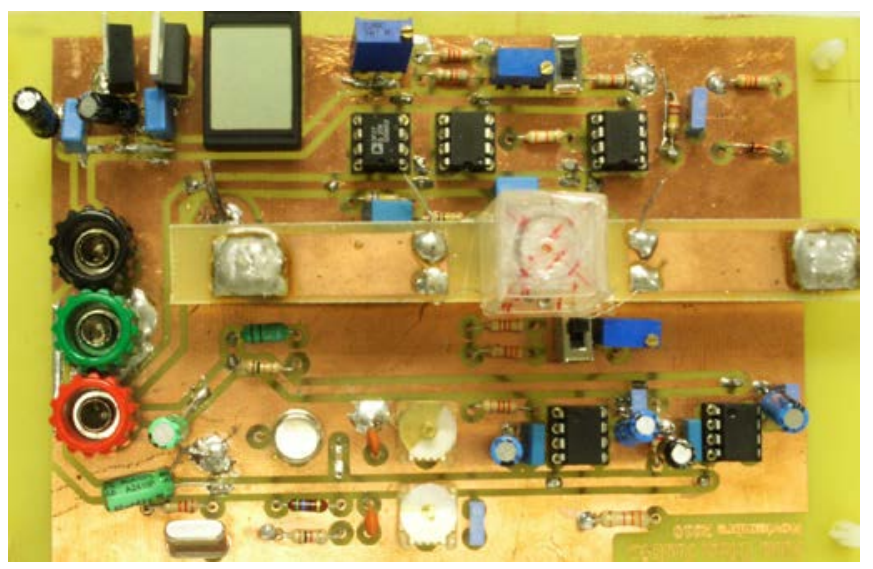

Fig. 14: Image of the signal conditioning circuit whose schematic is shown in Fig. 13.

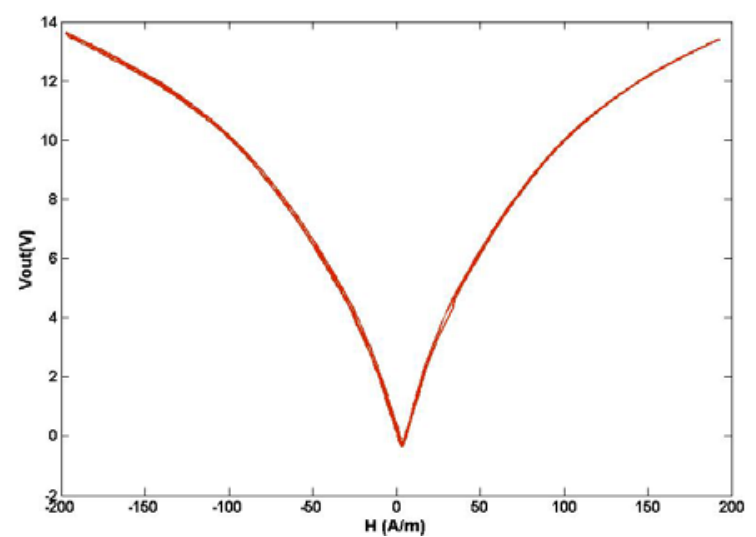

Fig. 15: Output voltage of the developed sensor as a function of the in-plane magnetic field.

\section{Conclusion}

We have designed and built an in-plane omnidirectional magnetic sensor based on a glass coated microwire. For this purpose, we have used a microwire with the shape of a circular loop. In order to model the sensor response, a simplified approach for the calculation of the impedance of a circular microwire is presented. This model is based on the sum of the contributions of small pieces of microwire with different angles with respect to the direction of the incident magnetic field. It is worth to note that this method allows the calculation of microwires with any shape, which paves the way to new sensor designs based on the shape of the microwire.

We have designed and built a signal conditioning circuit for the measurement of in-plane magnetic fields. The sensor presents a monotonic response in the range $\pm 200 \mathrm{~A} / \mathrm{m}$ which can be easily linearized with a digital controller.

The extension to an omnidirectional sensor would consist of two of these circular loops placed perpendicularly, each one sensing the component of magnetic field laying in the plane of the loop.

\section{Acknowledgements}

Hector García-Miquel is grateful to the Universitat Politècnica de València for the concession of a sabbatical year to have a research stay at the Cavendish Laboratory, and also want to express his gratitude to the Govern de la Generalitat Valenciana for financial support for this research stay at the Cavendish laboratory.

\section{References}

[1] D.-X. Chen, J. L. Muñoz, A. Hernando, and M. Vázquez. Magnetoimpedance of metallic ferromagnetic wires, Phys. Rev. B 57 (1998) 10699-10704.

[2] B. Dufay, S. Saez, C. Dolabdjian, A. Yelon, D. Menard, Physical properties and giant magnetoimpedance sensitivity of rapidly solidified magnetic microwires, J. Magn. Magn. Mater. 324 (2012) 2091-2099.

[3] M. Knobel, K.R. Pirota, Giant magnetoimpedance: concepts and recent progress, J. Magn. Magn. Mater. 242-245 (2002) 33-40.

[4] K.R. Pirota, L. Kraus, H. Chiriac, M. Knobel, Magnetic properties and giant magnetoimpedance in a CoFeSiB glass-covered microwire, J. Magn. Magn. Mater. 221 (2000) 243-247.

[5] L.V. Panina, D.P. Makhnovskiy, K. Mohri, Magnetoimpedance in amorphous wires and multifunctional applications: from sensors to tunable artificial mi- crowave materials, J. Magn. Magn. Mater. 272 (2004) 1452-1459.

[6] G.V. Kurlyandskaya, D. de Cos, S.O. Volchkov, Magnetosensitive transducers for nondestructive testing operating on the basis of the giant magnetoipedance effect: a review, Russ. J. Non-Destr. Test. 45 (2009) 377-398.

[7] A. Zhukov, M. Ipatov, J. Gonzalez, JM Blanco, V. Zhukova. Recent advances in studies of magnetically soft amorphous microwires. J. Magn. Magn. Mater. 321 (2009) 822-825.

[8] M. Vázquez, JM García-Beneytez, JM García, JP Sinnecker, A Zhukov. Giant magneto-impedance in heterogeneous microwires. J. Magn. Magn. Mater. 11 (2000) 6501-6505. 
[9] KR Pirota, L Kraus, H Chiriac, M Knobel. Magnetic properties and giant magnetoimpedance in $\mathrm{CoFeSiB}$ glass-covered microwire. J. Magn. Magn. Mater. 221 (2000) L243-L247.

[10] H. Garcia-Miquel, M. Vazquez, Ferromagnetic resonance in Corich glass-coated amorphous microwires, Physica B 299 (2001) 225-229.

[11] H. Garcia-Miquel, M.J. Esbri, J.M. Andres, J.M. Garcia, J.M. GarciaBeneytez, M. Vazquez, Power absorption and ferromagnetic resonance in Co-rich metallic glasses, IEEE Trans. Magn. 37 (2001) 561-564.

[12] S. E. Lofland, H. García-Miquel, M. Vázquez, S. M. Bragat. Microwave magnetoabsortion in glasscoated amorphous microwires with radii close to skin depth. J. Appl. Phys. 92 (2002).

[13] A. Hernando, V. Lopez-Dominguez, E. Ricciardi, K. Osiak, P. Marin. Tuned scattering of Electromagnetic Waves by a Finite Length Ferromagnetic Microwire. IEEE Trans. on Ant. and Prop. [0018-926X] vol.64 (3), 1112 -1115, 2016

[14] JM Garcia-Beneytez, F Vinai, L Brunetti, H Garcia-Miquel, M Vazquez. Study of magneto impedance effect in the microwave frequency range for soft magnetic wires and microwires. Sens. Actuators, A vol.81, 1-3 (2000) 78-81.

[15] H. Garcia-Miquel, J. Carbonell, J. Sanchez-Dehesa. Modulation of electromagnic waves by alternating currents through left-handed ferromagnetic microwires. J. Appl. Phys. 111 (2012) 063901.

[16] S.M. Bhagat, H. Garcia-Miquel, S.E. Lofland, Ferromagnetic resonance and antiresonance in glass-coated amorphous microwires, J. Magn. Magn. Mater. 249 (2002) 274-277.

[17] H. Garcia-Miquel, J. Carbonell, J. Sanchez-Dehesa., Left handed material based on amorphous ferromagnetic microwires tunable by dc current, Appl. Phys. Lett. 97 (2010) 094102.

[18] El Kammouni, M. Vázquez, L. Lezama, G. Kurliandskaya, L. Kraus. Temperature dependence of microwave absorption phenomena in single and biphase soft magnetic microwires. J. Magn. Magn. Mater. 368 (2014) 126-132.

[19] Y Luo, HX Peng, FX Qin, M Ipatov, V Zhukova, A Zhukov, J Gonzalez. Metacomposite characteristics and their influential factors of polymer composites containing orthogonal ferromagnetic microwire arrays. J. Appl. Phys. 115 (2014) 173909.

[20] N.A. Usov, A.S. Antonov, A.N. Lagar'kov, Theory of giant magneto-impedance effect in amorphous wires with different types of magnetic anisotropy, J. Magn. Magn. Mater. 185 (1998) 159-173.

[21] L. Kraus. Theory of giant magneto-impedance in the planar conductor with uniaxial magnetic anisotropy. J. Magn. Magn. Mater. 195 (1999) 764-778.

[22] L.V. Panina, K. Mohri, Magneto-impedance effect in amorphous wires, Appl.Phys. Lett. 65 (1994) 1189-1191.

[23] H. Garcia-Miquel, JM Garcia, JM Garcia-Beneytez, M Vazquez. Surface magnetic anisotropy in glass-coated amorphous microwires as determined from ferromagnetic resonance measurements. J. Magn. Magn. Mater. 231 (2001) 38-44.

[24] V.M. García-Chocano, H. García-Miquel. DC and AC linear magnetic field sensor based on glass coated amorphous microwires with Giant Magnetoimpedance. J. Magn. Magn. Mater. 378 (2015) 485-492.

[25] L.V. Panina, K. Mohri, K. Bushida, M. Noda, Giant magnetoimpedance and magneto-inductive effects in amorphous-alloys, J. Appl. Phys. 76 (1994) 6198-6203.

[26] K. Mohri, L.V. Panina, T. Uchiyama, K. Bushida, M. Noda, Sensitive and quick response micro magnetic sensor utilizing magnetoimpedance in Co-rich amorphous wires, IEEE Trans. Magn. 31 (1995) 1266-1275.

[27] H. Chiriac, M. Tibu, A.E. Moga, D.D. Herea, Magnetic GMI sensor for detection of biomolecules, J. Magn. Magn. Mater. 293 (2005) 671-676.

[28] Bing Han, Tao Zhang, Dongyan Huang, Xinglong Yue, Yu Zhou, Mingshu Bi, Giant magnetoimpedance current sensor with spiral structure double-probe, IEEE Trans. Magn. 45 (2009) 1999-2002.

[29] K. Mohri, T. Uchiyama, L.P. Shen, C.M. Cai, L.V. Panina, Sensitive micro mag- netic sensor family utilizing magneto-impedance (MI) and stress-impedance (SI) effects for intelligent measurements and controls, Sens. \& Actuators A 91 (2001) 85-90.
[30] T. Uchiyama, K. Mohri, Y. Honkura, L.V. Panina, Recent advances of pico-tesla resolution magneto-impedance sensor based on amorphous wire CMOS IC MI sensor, IEEE Trans. Magn. 48 (2012) 3833-3839.

[31] A. S. Antonov, N. A. Buznikov, A. B. Granovsky. Asymmetric Giant Magnetoimpedance of Amorphous Microwires under the Action of Torsional Stresses. J. Magn. Magn. Mater. 355 (2014) 289-294.

[32] D. P. Makhnovskiy, L. V. Panina, and D. J. Mapps. Field-dependent surface impedance tensor in amorphous wires with two types of magnetic anisotropy: Helical and circumferential. P. Rev. B, 63 (2001) pp.144424

[33] N.A. Buznikov, A.S. Antonov, A.B. Granovsky, C.G. Kim, C.O. Kim, X.P. $\mathrm{Li}$, S.S. Yoon. Current distribution and giant magnetoimpedance in composite wires with helical magnetic anisotropy. J. Magn. Magn. Mater. 296 (2006) 77-88. 